\title{
Study of the Photon Flux and the Dose Rate in the Vicinity of a ${ }^{60} \mathrm{Co}$ Gamma Irradiator
}

\author{
Elassaad Jemii ${ }^{1,2 *}$, Lotfi Ghedira ${ }^{1}$ \\ ${ }^{1}$ Physics Department, Faculty of Science, University of Monastir, Monasrir, Tunisia \\ ${ }^{2}$ Physics Department, College of Science in Zulfi, Al Majmaah University, Az-Zulfi, Saudi Arabia \\ Email: "elassaad_fsm@yahoo.fr
}

Received 3 March 2016; accepted 5 April 2016; published 8 April 2016

Copyright (C) 2016 by authors and Scientific Research Publishing Inc.

This work is licensed under the Creative Commons Attribution International License (CC BY).

http://creativecommons.org/licenses/by/4.0/

c) (i) Open Access

\begin{abstract}
The present work presents an overview of the study of some dosimetric quantities in the vicinity of the Tunisian Gamma Irradiation Facility. Firstly, we have confirmed our previous calculation of the photon flux and the dose rates, using a simulation with GEANT 4. A good agreement between calculation and simulation was obtained, which well confirmed the modeling of the CNSTN extended source by a pencil-like source. Secondly we have determined the isodose curves in the vicinity of the irradiator using a straightforward calculation. Finally, we have presented many comments for some published work concerning the methods used to determine these dosimetric quantities.
\end{abstract}

\section{Keywords}

Photon Flux, Dose Rates, Simulation GEANT 4, Isodose Curves

\section{Introduction}

The irradiator type SV68 installed in the Tunisian National Center for Nuclear Science and Technology (CNSTN) is an extended gamma source. It is designed for medical devices sterilization and foodstuff preservation [1]. It consists of eight ${ }^{60} \mathrm{Co}$ pencils like-sources doubly encapsulated in welded stainless-steel and zirconium layers.

The eight pencils of $1 \mathrm{~cm}$ diameter and $45 \mathrm{~cm}$ height [2], are arranged in two levels. Four pencils are mounted around a $7 \mathrm{~cm}$ radius circle, upward along the $\mathrm{Z}$ axis. The other four are mounted around a $4 \mathrm{~cm}$ radius circle, downward the $\mathrm{Z}$ axis (Figure 1). The source rack contains 10 free housings allowing the addition of supplementary pencils in order to compensate the decrease of the activity [3]. The total source activity in August/2004 is about $1.81 \mathrm{PBq}[5]$.

"Corresponding author. 


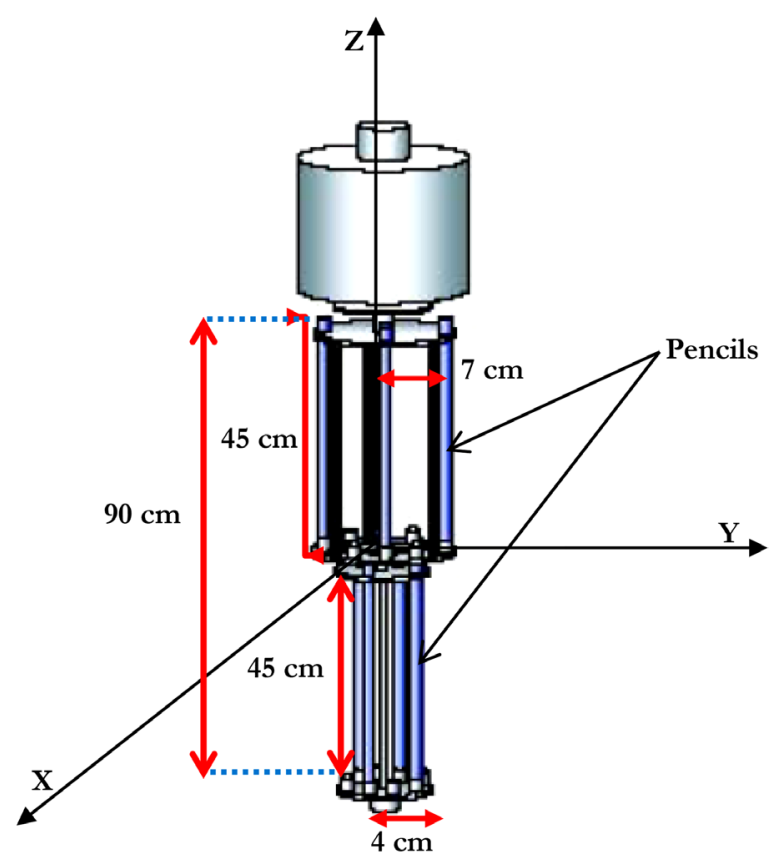

Figure 1. The CNSTN extended source in the irradiation position.

In order to determine the photon flux rates and the dose rates deposited in PMMA dosimeters placed in the vicinity of this irradiator, various measurements have been realized by the CNSTN researchers. These authors have also determined these two quantities using a calculation [3] [4] and a simulation by GEANT4 [5]-[8]. Their photon flux rates calculation is based on a multipole expansion. For the dose rate simulation, they simulated the PMMA dosimeters by spheres filled with water. These authors assumed that the photons flux rate is proportional to the dose rate deposited in such dosimeters.

In the present work, we will present how to calculate these quantities and we will discuss some results obtained by these authors.

\section{Photon Flux}

In a previous works [2] [9], we have Shown, using a straightforward calculation of the photon flux, that The CNSTN irradiator can be modeled by a single pencil with the same activity and the same height. Thus, the expression of the photon flux rates, intercepting a PMMA dosimeter placed in the vicinity of a pencil-like source is given by:

$$
\varphi_{\gamma}(\boldsymbol{r})=\frac{A}{8 \pi L X^{2}} \int_{-L}^{L} \sin \theta^{\prime 3} \mathrm{~d} z
$$

where $A$ is the source activity, $L=45 \mathrm{~cm}$ is the length of the pencil and $X$ is the position of the vertical plan contain the dosimeters. For more confirmation, we run the GEANT 4 code, in order to compare the simulated results with our previous calculation. Figure 2 shows the comparison between the calculation and simulation the photon flux at four vertical plans. A very good absolute agreement is obtained. This result confirms that the CNSTN extended source is equivalent to a single pencil with the same activity and the same height.

\section{Dose Rates}

In the previous work [9], we also have determined the expression of the dose rates deposited in a PMMA dosimeter placed in the vicinity of a pencil-like source. To calculate the dose rate, we have used the following expression:

$$
d(\boldsymbol{r})=2 \frac{1}{m_{d}} \frac{\mathrm{d} N_{e}}{\mathrm{~d} t}(\boldsymbol{r}) \bar{E}_{C}^{\prime}
$$




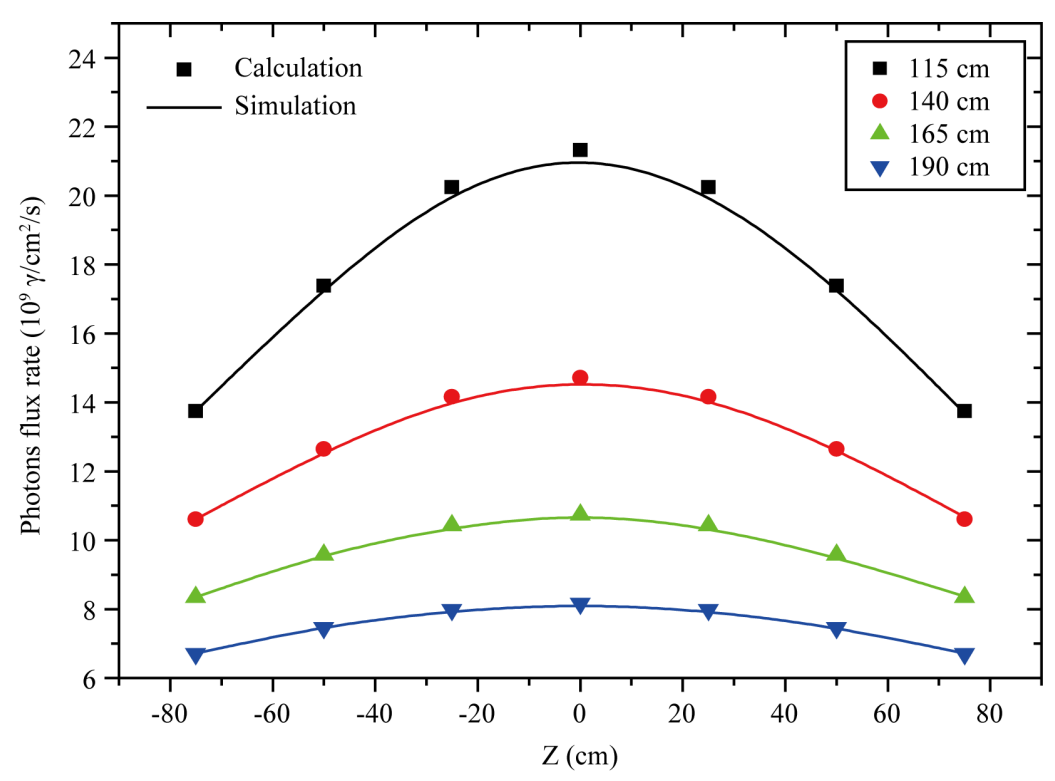

Figure 2. Calculated and simulated photon flux rates at for vertical plans.

where the factor 2 corresponds to the two ${ }^{60}$ Co photons (1.17 and $\left.1.33 \mathrm{MeV}\right), \bar{E}_{C}^{\prime}$ is the mean energy loss in a PMMA dosimeter, $m_{d}$ is the dosimeter mass and $\frac{\mathrm{d} N_{e}}{\mathrm{~d} t}$ is the number of electrons per unit time [9].

After integration, we have obtained a simple expression given by:

$$
d(X, Z)=K \frac{1}{X}\left(\operatorname{arctg}\left(\frac{Z+L}{X}\right)-\operatorname{arctg}\left(\frac{Z-L}{X}\right)\right) f(X, Z)
$$

where $f(X, Z)$ is the correction factor due to escaping effect of the Compton-electrons and $K$ is a constant.

To confirm this result, we also run the code GEANT 4 . The dose rate is determined by transforming electrons deposited energy inside the PMMA dosimeter of mass $m$ during a run of $N$ events. The dose rates formula is given by:

$$
\frac{\mathrm{d} D}{\mathrm{~d} t}(\mathrm{~Gy} / \mathrm{h})=2 \frac{E_{d}(\mathrm{MeV}) \times A(\mathrm{~Bq}) \times C}{N \times m(\mathrm{~g})}
$$

where $A$ is the source activity $(1.81 \mathrm{PBq})$, the factor 2 corresponds to the two ${ }^{60}$ Co photons, $E_{d}$ is the total energy deposited in a dosimeter and the coefficient $C$ represents the conversion factor from $\mathrm{MeV} / \mathrm{g}$ to $\mathrm{Gy} / \mathrm{h} C=(1.6 \times$ $\left.10^{-10} \times 3600\right) \mathrm{Gy} / \mathrm{h}[7]$.

Figure 3 shows the simulated and calculated absolute dose rates in PMMA dosimeters placed in a vertical plan at $150 \mathrm{~cm}$ from the source. A very good agreement is obtained which represents another proof that the CNSTN extended source can be modeled by a single pencil.

Note that the researchers of the CNSTN have assumed that the dose rate deposited in a PMMA dosimeter placed in the vicinity of the irradiator is proportional to the photon flux intercepting this dosimeter which is, unfortunately, not true.

To confirm the non-proportionality, between these two quantities, we have used a simulation by GEANT 4 . Figure 4 shows the simulated relative photon flux rates and the simulated relative dose rates in a PMMA dosimeter at $190 \mathrm{~cm}$ from the source. It is clear that the two quantities are not proportional.

\section{Isodose Curves}

The isodose curves represent the set of points in space receiving the same dose at the same irradiation conditions. The same authors [4] have determined the isodose curves, using their multipole expansion. They calculated the isodose curves in a vertical plan at $40 \mathrm{~cm}$ from the irradiator. We already showed that this multipole expansion 


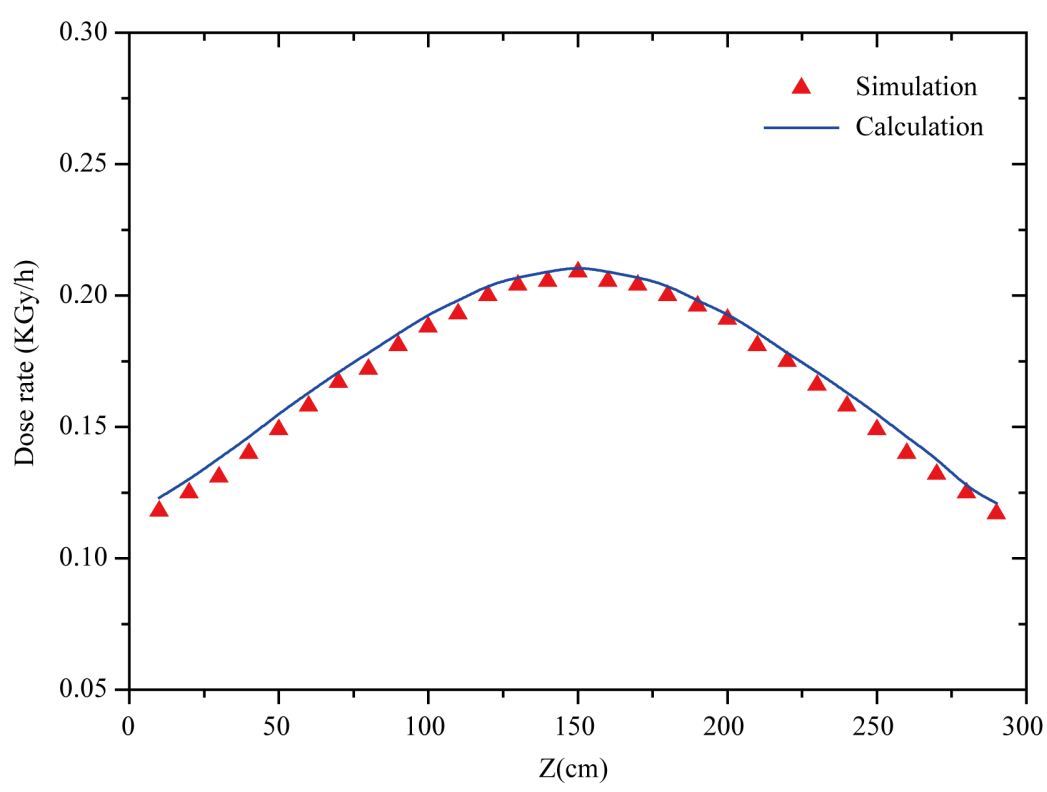

Figure 3. Calculated and simulated dose rates along a vertical axis at $X=150 \mathrm{~cm}$.

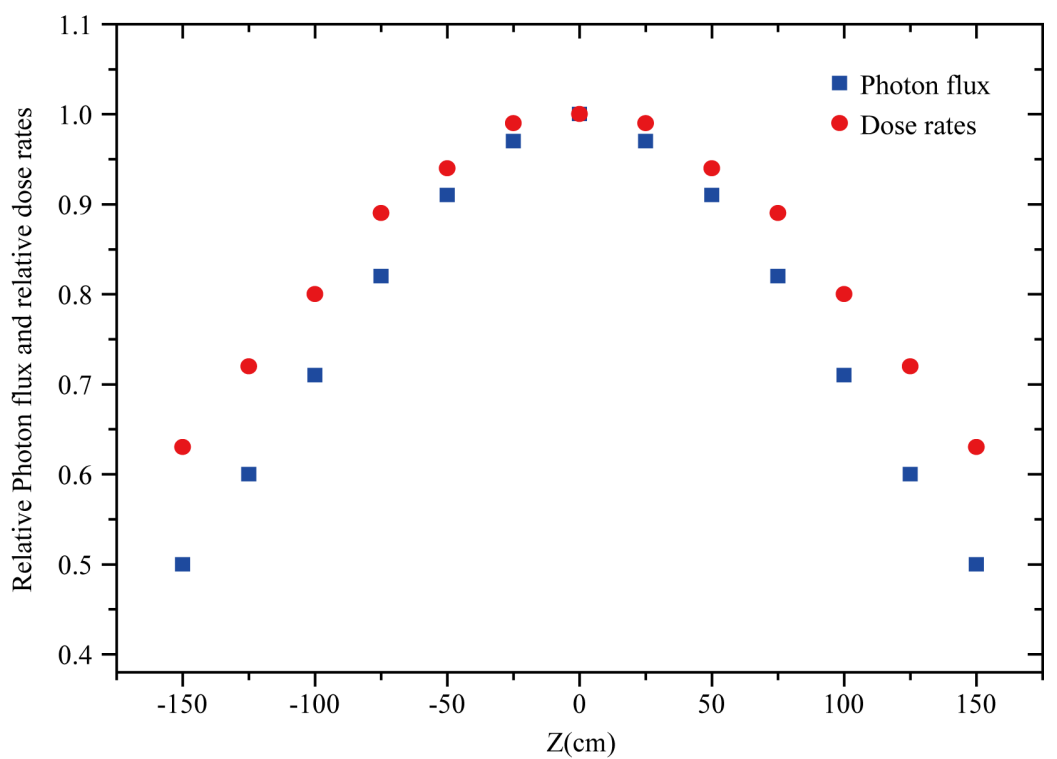

Figure 4. Relative photon flux rates and relative dose rates at $X=190 \mathrm{~cm}$.

is useless in our case, so the dose mapping found by this author is questionable. We have determined these isodose curves using the equation of the dose rate found previously (Equation (3)).

The isodose curves are then given by the following equation: $d(X, Z)=$ cte. Figure 5 shows some curves represents an absolute dose map at four different plans $(X=50,100,150$ and $200 \mathrm{~cm})$. The unit of the dose rate in the following figure is $\mathrm{Gy} / \mathrm{h}$. It is clear that the isodose curves present an elliptical shape.

\section{Comments}

1) Concerning the calculation of the photon flux, some authors [3] [4] [10] have used a multipole expansion as a function of $\frac{r^{\prime}}{r}$, where $\boldsymbol{r}^{\prime}$ is the vector position of an elementary source activity and $\boldsymbol{r}$ is the vector position of the dosimeter relative to the source (Figure 1 in Ref. [3]). 


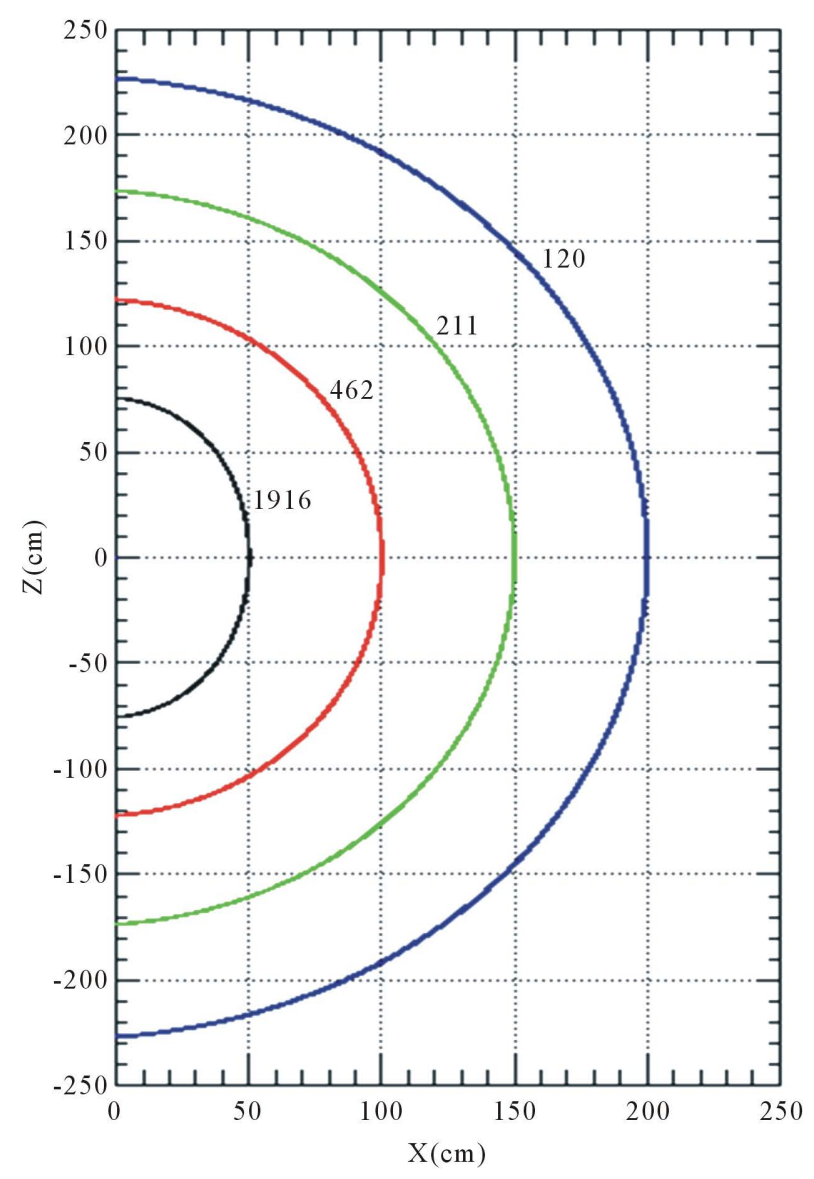

Figure 5. Isodose curves at four vertical plans.

The expression used by these references was:

$$
\varphi(\boldsymbol{r})=\frac{K}{4 \pi r^{2}}\left[\int_{\Omega} P_{0}(\cos w) \rho_{A}\left(\boldsymbol{r}^{\prime}\right) \mathrm{d}^{3} \boldsymbol{r}^{\prime}+\frac{2}{r} \int_{\Omega} r^{\prime} P_{1}(\cos w) \rho_{A}\left(\boldsymbol{r}^{\prime}\right) \mathrm{d}^{3} \boldsymbol{r}+\frac{8}{3} \frac{1}{r^{2}}\left[\int_{\Omega} r^{\prime 2} P_{2}(\cos w) \rho_{A}\left(\boldsymbol{r}^{\prime}\right) \mathrm{d}^{3} \boldsymbol{r}^{\prime}+\cdots\right]\right]
$$

Note that this development is valid only if the distance between the source and the dosimeter is very large compared to the source dimensions. Given that the height of the CNSTN irradiator is $90 \mathrm{~cm}[2]$, the ratio $\frac{r^{\prime}}{r}$ can be, in some positions, even bigger than one $\left(r=40 \mathrm{~cm}\right.$ and $\left.r^{\prime}=45 \mathrm{~cm}\right)$.

In fact, if one has to use a multipole expansion, it must be a development in terms of $\frac{\rho}{r}$ which is always very small, $\rho$ being the mean radial extension of the CNSTN source $(\rho \approx 6 \mathrm{~cm})$.

Moreover, according to the calculation of these authors, the expression of the photon flux rate generated by a given gamma source is equal to: $\varphi(\boldsymbol{r})=\frac{K}{4 \pi} \int_{\Omega} \frac{\rho_{A}\left(\boldsymbol{r}^{\prime}\right) \mathrm{d}^{3} \boldsymbol{r}^{\prime}}{\left\lfloor\boldsymbol{r}-\boldsymbol{r}^{\prime}\right\rfloor^{2}}$, where $K$ is a constant. Thus, for a point-like source we obtain:

$$
\varphi(\boldsymbol{r})=K \frac{A}{4 \pi r^{2}}
$$

In our previous work [2], for a given point-like gamma source, the photon flux is given by $\varphi(\boldsymbol{r})=\frac{A}{4 \pi r^{2}} \sin \theta$, then the factor $\sin \theta$ has been omitted by these authors. Furthermore, the significance of the constant $K$ is not clear in these references, it has no reason to be added since it is clear that it is equal to 1. 


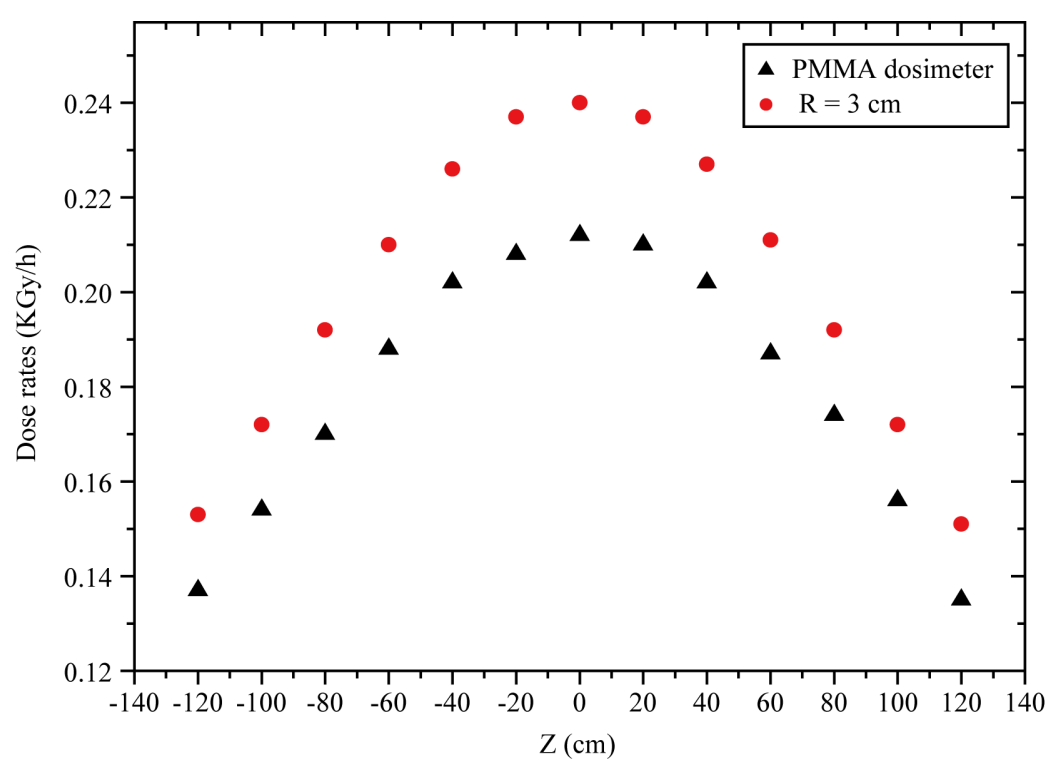

Figure 6. Simulated dose rates along the vertical axis for a $3 \mathrm{~cm}$ sphere radius and a PMMA dosimeter at $X=150 \mathrm{~cm}$.

2) To determine the dose rates deposited in a PMMA dosimeter, some authors [5]-[8] have used a spherical dosimeters instead of the PMMA dosimeters having a parallelepiped shape. According to these authors, the equivalent radius of the spheres is $4 \mathrm{~cm}$ in some cases and $3 \mathrm{~cm}$ in another case. Note that, for example the mass thickness of a $4 \mathrm{~cm}$ radius sphere is more than 12 times the range of forward Compton electrons. Thus the escaping effect is negligible which is not the case for the PMMA dosimeters (For $Z=0$ and $X=150 \mathrm{~cm}$, the escaping effect is about $20 \%$, it is clear that the escaping effect in a PMMA dosimeter is not negligible).

To show the difference, we have compared in Figure 6 the absolute simulated dose rates using PMMA dosimeters of $0.9 \mathrm{~cm}$ and spheres of $3 \mathrm{~cm}$ radius. A large discrepancy is obtained.

3) Some authors have determined the isodose curves, using their multipole expansion [4]. They calculated the isodose curves in a vertical plan at $40 \mathrm{~cm}$ from the irradiator and they give a relative absorbed dose map. We already showed that the multipole expansion cannot be used in our case, then all obtained results using this method are questionable.

4) The pencil holder of the CNSTN irradiator contains 10 free positions allowing the addition of other pencils in order to compensate the decrease of the activity. The reloading of the source can be done with or without permutation, which gives a large number of configurations (millions of configurations) [3]. We showed in our previous work [2] that this extended source behaves like a pencil source with a discrepancy less than one percent. This permutation is then useless since it is equivalent to a rotation of an axis, furthermore it is very expensive.

\section{Conclusion}

In this work, we have determined, using a simulation with GEANT 4, the photon flux and the dose rates in order to confirm that the CNSTN irradiator can be modeled by a pencil-like source. Secondly, we have underlined some mistakes made by the CNSTN researchers concerning the photon flux rates, the dose rates and the isodose curves. We have showed that, in our case (CNSTN Irradiator), the multipole expansion is useless to calculate the photon flux rate and the dose rates. Therefore, all obtained results based on this method are wrong. We have also showed that, in PMMA dosimeters, the photon flux rate is not proportional to the dose rate. The isodose curves in the vicinity of the CNSTN irradiator have been determined using a straightforward calculation. Finally, given that the CNSTN irradiator is an equivalent to a pencil-like source, we have underlined that the permutation of the pencils is useless.

\section{References}

[1] Farah, K., Barkallah, I., Benkraiem, H., et al. (2003) Radiation Dosimetry for Commissioning of Gamma Irradiation 
Irradiation Plant. Proceeding of the Sixth Arab Conference of the Peaceful Uses of Nuclear Energy IV, 125-143.

[2] Jemii, E., Mazouz, M., Ben Fred, A., et al. (2011) Modeling of the Tunisian ${ }^{60}$ Co Gamma Irradiator by a Coaxial Equal Height and Equal Activity Single Pencil. Radiation Physics and Chemistry, 80, 1158-1161. http://dx.doi.org/10.1016/j.radphyschem.2011.05.003

[3] Loussaief, A., Trabelsi, A. and Baccari, B. (2006) Extended Gamma Sources Modeling Using Multipole Expansion: Application to the Tunisian Gamma Source Load Planning. Radiation Physics and Chemistry, 75, 463-472. http://dx.doi.org/10.1016/j.radphyschem.2005.12.024

[4] Loussaief, A. and Trabelsi, A. (2007) Dose Mapping Using Multipole Moments. Nuclear Instruments and Methods in Physics Research A, Spectrometers, Detectors and Associated Equipment, 580, 102-105. http://dx.doi.org/10.1016/j.nima.2007.05.019

[5] Gharbi, F., Kadri, O. and Farah, K. (2005) Validation of GEANT 4 Code as Predictive Tool of Dose Rate Measurement in the Tunisian Gamma Irradiation Facility. Radiation Physics Chemistry, 74, 102-111. http://dx.doi.org/10.1016/j.radphyschem.2005.02.006

[6] Kardi, O., Gharbi, F. and Farah, K. (2005) Monte Carlo Improvement of the Dose Uniformity in Gamma Irradiation Processing Using the GEANT 4 Code. Nuclear Instruments and Methods in Physics Research B, 239, 391-398. http://dx.doi.org/10.1016/j.nimb.2005.05.052

[7] Kadri, O., Gharbi, F., Farah, K., et al. (2006) Monte Carlo Studies of the Tunisian Gamma Irradiation Facility Using GEANT 4 Code. Applied Radiation and Isotopes, 64, 170-177. http://dx.doi.org/10.1016/j.apradiso.2005.07.009

[8] Mannai, K., Trabelsi, A. and Maddouri, F. (2014) Angular Dependences of Response of Dosimeters Exposed to an Extended Radioactive Source. Radiation Physics Chemistry, 98, 51-56. http://dx.doi.org/10.1016/j.radphyschem.2014.01.007

[9] Jemii, E., Mazouz, M. and Ghedira, L. (2013) Dose Rate Calculation in the Vicinity of the Tunisian Gamma Irradiation. World Journal of Nuclear Science and Technology, 3, 28-32. http://dx.doi.org/10.4236/wjnst.2013.31005

[10] Loussaief, A., Mannai, K., Trabelsia, A., et al. (2007) Inner Radiation Field Modeling Using Multipole Moments. Radiation Physics and Chemistry, 76, 909-916. http://dx.doi.org/10.1016/j.radphyschem.2006.12.007 